\title{
Mobilidade em centros urbanos por circuitos de caminhada utilizando o método multicritério PROMETHEE, estudo de caso de Campinas
}

\author{
Mobility in urban center by walking circuits using the \\ PROMETHEE multicriteria method, Campinas case study
}

Marcius Fabius Carvalho [a] [D, Guilherme Kato Rodrigues [b] [D],

Cláudia Cotrim Pezzuto [a] [D], Lia Toledo Moreira Mota [a] (DD),

Marina Lavorato de Oliveira [a] [D]

[a] Universidade Católica de Campinas, Centro De Ciências Exatas, Ambientais e de Tecnologia (Ceatec), Campinas, SP, Brasil

[b] Universidade Católica de Campinas, Infraestrutura urbana, Campinas, SP, Brasil

Como citar: Carvalho, M. F., Rodrigues, G. K., Pezzuto, C. C., Mota, L. T. M. \& Oliveira, M. L. (2021). Mobilidade em centros urbanos por circuitos de caminhada utilizando o método multicritério PROMETHEE, estudo de caso de Campinas. urbe. Revista Brasileira de Gestão Urbana, v. 13, e20210087. https://doi.org/10.1590/2175-

3369.013.e20210087

\section{Resumo}

Este trabalho toma a caminhada como forma de mobilidade em um centro urbano e desenvolve uma metodologia para a determinação de rotas mais acessíveis aos pedestres que chegando aos terminais rodoviários urbanos desejem atingir pontos de interesse. Pretende-se com esta proposição oferecer melhores trajetos, para uma classe social vinda de bairros periféricos, de aceso a serviços essenciais. A proposta foi aplicada à região central de Campinas-SP, caracterizada por atividades comerciais e de prestação de serviços, atendida por dois importantes terminais públicos urbanos. Na determinação da melhor rota para caminhada foram utilizados três macros indicadores: Indicador de ambiente, representando características físicas do ambiente da caminhada; Indicador conforto, representado pela atratividade visual e conforto local; e Indicador segurança, representando a segurança pública e pessoal. A partir do método multicritério PROMETHEE, foram definidas as melhores rotas que conectam pontos de Origem (Terminal Urbano) a pontos de Destino no centro urbano de Campinas. A implantação destas rotas pelo poder público dotará o centro urbano de espaço para caminhada que atenda aos interesses do cidadão em três aspectos o ambiente, o conforto e a segurança.

Palavras-chave: Caminhabilidade. Caminhada. Mobilidade urbana. Métodos multicritérios. PROMETHEE.

MFC Professor Doutor do Programa de Infraestrutura urbana da Universidade Católica de Campinas, e-mail: marcius@puccampinas.edu.br

GKR Mestrando do Programa de Infraestrutura urbana da Universidade Católica de Campinas, e-mail: guilhermekatoo@gmail.com CCP Professora Doutora do Programa de Infraestrutura urbana da Universidade Católica de Campinas, e-mail: claupezzuto@gmail.com LTMM Professora Doutora do Programa de Infraestrutura urbana da Universidade Católica de Campinas, e-mail: lia.moreira.mota@gmail.com

MLO Professora Doutora do Programa de Infraestrutura urbana da Universidade Católica de Campinas, e-mail: mlavorato@gmail.com 


\section{Abstract}

This work takes walking as a form of mobility in an urban center and develops a methodology for determining more accessible routes for pedestrians who arrive at urban bus terminals and wish to reach points of interest. The proposal objective is to offer better routes, for a social class coming from peripheral neighborhoods, with access to essential services. The proposal was applied to the central region of Campinas-SP, characterized by commercial and service activities, served by two important urban public terminals. In determining the best walking route, three macros indicator were used: Environment indicator, representing physical characteristics of the walking environment; Comfort indicator, represented by visual attractiveness and local comfort; and Safety indicator representing public and personal security. Using the PROMETHEE multicriteria method, the best routes connecting points of Origin (Urban Terminal) to points of Destination in the urban center of Campinas were defined. The implementation of these routes by local government will provide the urban center with walking space that meets the interests of the citizen in three aspects: the environment, comfort, and safety.

Keywords: Walkability. Walking. Urban mobility. Multicriteria methods. PROMETHEE.

\section{Introdução}

O Brasil é marcado por profundas desigualdades evidenciadas por enorme diversidade socioespacial como consequência de um desenvolvimento urbano ocorrendo desacoplado do desenvolvimento econômico e social. Com isto, restou aos setores de menor renda a alternativa dos loteamentos ou conjuntos habitacionais periféricos, onde se encontravam terrenos de mais baixos valores, longe do trabalho, da assistência à saúde e ao lazer (BRASIL, 2012).

Os centros urbanos, esvaziados de moradias, tornam-se centros econômicos e de serviços, com a mobilidade privilegiando o transporte viário individual ou mesmo coletivo, que reproduzem modelos insustentáveis do ponto de vista ambiental e socioeconômico, desconsiderando a qualidade de vida de seus habitantes (PLANMOB, 2015). 0 cidadão em sua maioria passa a convergir ao centro da cidade por terminais públicos, à busca de trocas comerciais e de serviços públicos essenciais concentrados neste espaço.

A presença do transporte coletivo ou individual no centro urbano sobrecarrega ruas e avenidas, dificulta a circulação de pessoas, prejudica o comércio local, contribui para a poluição ambiental e sonora e desincentiva o cidadão a permanecer neste meio caótico. Diversas pesquisas propõem soluções para mitigar os efeitos negativos destes centros, sendo reconhecida por elas a importância da caminhada (Clifton et al., 2016).

O Plano Nacional de Mobilidade Urbana (PLANMOB, 2015) afirma que a mobilidade urbana interfere diretamente na vida do cidadão, pois é responsável pela circulação de pessoas e de cargas nas cidades, influenciando diretamente o desenvolvimento econômico local, a logística para distribuição de produtos, a saúde e a produtividade da população. E define a caminhada como a ação de deslocamento de uma pessoa pelo esforço próprio, sem o uso de sistema motorizado ou bicicletas, partindo da origem até o destino da viagem, e podendo ser um modo integrador com outros modais (PLANMOB, 2015).

Dados da Associação Nacional de Transporte Público (ANTP, 2018), indicam que 39,2\% das viagens diárias em cidades com mais de 60.000 habitantes, são realizadas exclusivamente a pé. Este é um fator motivador para que o espaço urbano seja voltado ao bem-estar do pedestre e equidade no uso do espaço público de circulação, vias e logradouros (BRASIL, 2012).

No sentido de atingir a mobilidade sustentável em centros urbanos, pesquisas são realizadas, principalmente considerando a caminhada como o modo mais sustentável (Bivina \& Parida, 2019; Carvalho, 2018; Silva \& Angelis Neto, 2019; Malatest, 2007; Magagnin, 2009; Nag et al., 2020; Oliveira, 2014; PLANMOB, 2015; Velozo, 2019; e Yamamoto, 2017). De forma geral, estes trabalhos destacam a importância de ambiente de caminhada seguro e confortável e estabelecem diretrizes para os planejadores desenvolverem projetos adequados para um centro urbano. 


\section{Mobilidade Urbana}

Conforme a Lei 12.587 de 2012 da Política Nacional de Mobilidade Urbana, a mobilidade urbana é tema amplo, que abrange não apenas a questão do transporte de cargas e pessoas, como também questões de desenvolvimento urbano como o uso e a ocupação do solo, a saúde e a qualidade de vida das pessoas nas suas necessidades de locomoção no cotidiano (BRASIL, 2012). Pode ser associada à necessidade de movimentação do cidadão em um espaço, seja qual for sua condição, de pedestre, motorista, ciclista ou passageiro, o que necessita de boas condições independentemente do modo de locomoção. A Lei 10.257 de 2001 denominada Estatuto da Cidade estabelece a garantia do direito à cidade como um dos direitos fundamentais do cidadão (BRASIL, 2001).

O PLANMOB (2015) afirma que a questão da mobilidade envolve aspectos ambientais, econômicos e sociais, portanto tem carácter multidimensional, o que a torna de difícil solução nas grandes áreas urbanas. No entanto, acentua a necessidade da encontrar uma solução embasada nas melhores práticas e legislações que busque formas mais limpas, energeticamente eficientes e adequadas aos principais interessados.

Este trabalho considera a caminhada como a forma de priorizar a saúde, a questão ambiental e sonora, e menor dependência dos meios motorizados (seja individual ou coletivo). Para tanto, propõe o estabelecimento de rotas para caminhada baseadas em indicadores associados ao ambiente, a segurança e ao conforto do pedestre.

\section{A caminhada}

A caminhada ou modo a pé é a ação de deslocamento resultante do esforço próprio, sem o uso de sistema motorizado ou bicicletas, por meio do viário disponível como passeios, calçadas, calçadões, passarelas, entre outros, desde uma origem até um destino, ou o meio de transporte para integração com outros modais, como ônibus, veículos e bicicleta a um ponto de destino (PLANMOB, 2015).

O caminhar é o principal modo de mobilidade do ser humano no mundo e o mais acessível, pois é o mais barato e requer menores recursos de infraestrutura Asadi-Shekari et al. (2014). Faz parte de uma vida saudável, amenizando os efeitos negativos das externalidades da vida moderna tais como sobrepeso e obesidade. Vários estudos sobre saúde e planejamento urbano apontam que a existência de infraestrutura adequada tem relação direta com as taxas de caminhada portanto, com uma vida mais saudável (Pucher et al., 2010; Asadi-Shekari et al. 2019). Ao mesmo tempo, a caminhada exerce influência sobre outras externalidades negativas associadas às viagens motorizadas como consumo de combustível, poluição do ar e sonora e problemas de segurança. É também um meio mais acessível de mobilidade, por ser mais barato e requerer infraestrutura mais simples.

A somatória de fatores contendo áreas com maior densidade de atividades, áreas com uma maior diferenciação do uso do solo compostas de serviços públicos e privados, residências e comércio e áreas com um desenho urbano de qualidade são as mais favoráveis ao desenvolvimento e à prática da caminhada (Valentini, Kuhn \& Vargas, 2017; Tian \& Ewing, 2017). Estes motivadores associados a fatores do ambiente construído como percepções dos pedestres, nível de serviço das calçadas influenciam na opção pela mobilidade a pé.

A caminhada é a única atividade de mobilidade que não produz emissão, está sempre disponível, não requer nenhum equipamento, é segura e sem custos (Ernawati, 2016). Entretanto, a adesão a ela depende da distância a ser percorrida, infraestrutura adequada, estética do bairro, atividades comerciais e segurança no percurso e pode ser inserida no conceito de "a vida na cidade acontece a pé (Carvalho, 2018). Reconhecendo que a decisão de escolha pela caminhada está relacionada com as motivações e as barreiras de cada indivíduo, Krizek, Forsyth e Baum (2009) afirmam que são necessárias políticas e infraestrutura para tornar a caminhada atraente. 


\section{Fatores que influenciam na mobilidade por caminhada}

Da Silva e Angelis Neto (2019) propuseram um método de avaliação do nível de serviço das calçadas denominado ISC (Índice de Serviço das Calçadas), que faz uma análise técnica das calçadas e coleta da percepção dos pedestres por meio de questionários. O ISC considera a importância relativa de cada parâmetro para a atribuição dos níveis de serviço de cada segmento de calçada a partir de um sistema de escores representado entre 0 e 5 ( 0 como o pior caso, e 5 como o melhor). 0 ISC é dividido em dois grupos com cinco critérios cada, o primeiro grupo definido pela qualidade do espaço e analisa as condições de segurança, o segundo grupo considera a acessibilidade. 0 cálculo é feito para cada quadra, segmento de caminhada, por meio de uma média dos valores dos dois grupos (Silva \& Angelis Neto, 2019).

Carvalho (2018) desenvolveu um índice de caminhabilidade para avaliar o ambiente construído, sob a perspectiva do pedestre que na maioria das vezes é subjetiva, transformando-a em números, dados objetivos que auxiliem em uma tomada de decisão pelo gestor público. A autora avalia o ambiente de caminhada por oito categorias: acessibilidade, atratividade, conectividade, conforto, segurança pública, segurança viária, uso do solo, e travessia, divididas em 27 indicadores sendo 24 referentes aos segmentos de calçada e 3 para análise de travessias. Os indicadores são resultados de respostas a questionário referente ao indicador, com as respostas variando de 1 até 5 , sendo 1 a melhor nota.

Silva e Angelis Neto (2019), e Carvalho (2018) fazem uma média final de todos os indicadores para determinação do Índice de caminhabilidade para cada quadra. Entretanto, a autora ao contrário que propõe os outros autores faz uma normalização dos valores para ajustá-los em uma mesma escala, variando entre 0 a 100. Para a autora, zero é o maior impacto ou melhor satisfação, e 100 é o pior impacto ou pior satisfação (Carvalho, 2018). A pesquisa de Carvalho (2018) tomou como avaliação final a média aritmética das categorias, ou seja, de todos os indicadores, o que faz com que um atributo melhor qualificado compense um pior na análise da caminhabilidade. Deve-se destacar que em muitos casos esta forma de cálculo não reflete a realidade.

O Instituto de Políticas de Transporte \& Desenvolvimento (ITDP BRASIL, 2018) desenvolveu um índice de caminhabilidade composto por 6 categorias: calçada, mobilidade, atração, segurança viária, segurança pública e ambiente, que foram divididos em outros 15 indicadores.

Assim como para Carvalho (2018), e Silva e Angelis Neto (2019), o ITDP BRASIL (2018) também avalia os indicadores por segmento de quadra, dividindo a rua em dois lados de calçadas, portanto, considerando o mesmo trecho de calçada com duas classificações distintas. A metodologia para cada indicador consiste em uma pontuação entre 0 a 3 (sendo 0 insuficiente e 3 como ótimo), obtendo uma média aritmética simples para cada categoria, com seus respectivos indicadores. Considera que quanto mais extenso o segmento de calçada maior deve ser seu peso na pontuação final de cada categoria, portanto, para o método do ITDP BRASIL (2018), há uma ponderação maior quando a extensão do segmento for maior.

Bivina e Parida (2019) consideram que a decisão de caminhar passa por uma análise de quatro principais critérios: Segurança Pessoal, Segurança Geral, Conforto e Conveniência, e Mobilidade e Infraestrutura, dividida em 17 subcritérios. As autoras utilizam o AHP (Analytical Hierarchy Process), método multicritério de tomada de decisão, com os pesos atribuídos a cada critério pela aplicação de questionários aos pedestres. Os resultados do estudo apontam para existência de uma diferença significativa na prioridade para várias necessidades em medidas de ambiente construído de caminhada com base no sexo e faixa etária e os pedestres percebem a segurança e a proteção como indicadores mais críticos que a mobilidade. Entretanto, somente a avaliação do pedestre não é suficiente para o melhor planejamento para a caminhada, pois a maioria dos entrevistados não possuem deficiências, não veem importância neste requisito, o que leva a uma ponderação irrisória para este indicador.

Nag et al., (2020) em seu estudo de revisão sobre o nível de serviço de pedestres (PLOS - Pedestrian Level of Service), encontraram 389 subcritérios com frequência de conceitos duplicados e/ou com terminologia inconsistente, e apontaram que 46 dos 47 estudos escolheram os atributos sem rigor para avaliar o ambiente de caminhada. Os autores, como Bloomberg \& Burden (2006), pontuam que a percepção 
do pedestre desempenha papel importante na decisão de caminhar, influenciada por três principais indicadores: Ambiente Construído, Característica do Fluxo, e Percepção dos Usuários.

Nenhum dos estudos de Nível de Serviço dos usuários faz referência a "rede de pedestres", ou seja, da conectividade das rotas dos pedestres não foram considerados na maioria dos trabalhos de revisão, o que pode prejudicar a avaliação da opção de caminhada no ambiente construído (Nag et al., 2020).

A proposta deste trabalho toma a caminhada como a forma de mobilidade adequada para curtas distâncias e propõe uma metodologia para seleção de rotas entre origens e destinos de um centro urbano. Tem o caráter social ao considerar que estas rotas de caminhada conectam pontos de origem (terminais urbanos centrais) a pontos de atendimento de serviços públicos que atendam a necessidade daquele cidadão residente na periferia. A pesquisa é inovadora por partir da identificação e aplicação de indicadores que influenciam na decisão por caminhar e propor que o centro urbano seja planejado com rotas de caminhada entre pontos de interesses, considerados como terminais urbanos, e pontos de atratividade principalmente constituídos de serviços públicos essenciais em um centro urbano, atendendo uma questão social. A escolha da melhor rota para caminhada para um centro urbano é tratada como um problema multicritério por meio do método PROMETHEE II e o software gratuito Visual PROMETHEE (VPSOLUTIONS, 2015).

\section{Metodologia}

Esta seção foi estruturada nos seguintes itens: delimitação da área de estudo, matriz origem destino, seleção de indicadores, matriz segmento indicador, desempenho das rotas e Método multicritério PROMETHEE.

\section{Delimitação da área de estudo e especificação dos pontos de interesse}

Foi escolhida uma região central da cidade de Campinas no Estado de São Paulo situada a noroeste da capital como estudo de caso. Campinas é a $3^{\underline{a}}$ maior cidade do estado de São Paulo, atrás de Guarulhos (2 ${ }^{a}$ ) e da capital São Paulo (1 $1^{a}$ ) e a $14^{a}$ maior cidade do Brasil (IBGE CIDADES, 2010).

A área de estudo foi tomada como um recorte da região central da cidade de Campinas, considerando sua elevada importância comercial e de prestação de serviço. Consta deste recorte dois grandes terminais públicos urbanos (Terminal Central (PO1) e Terminal Mercado (PO2)) e diversos pontos de atratividade (PD1, PD2, PD3 e PD4), principalmente aqueles relacionados à prestação de serviços públicos, que requerem atenção com relação às questões de mobilidade na região, Figura 1. Estes pontos estão descritos na Tabela 1 e Figura 1.

O Terminal Central (P01) é o maior e principal terminal da cidade de Campinas, atende cerca de 70 mil passageiros por dia, e recebe 32 linhas de ônibus (EMDEC, 2010a). 0 Terminal Mercado (PO2) foi o primeiro ponto da cidade a estruturar-se como um terminal, foi inaugurado no final da década de 70, atualmente atende cerca de 20 mil passageiros por dia, recebe 28 linhas de ônibus (EMDEC, 2010b). 


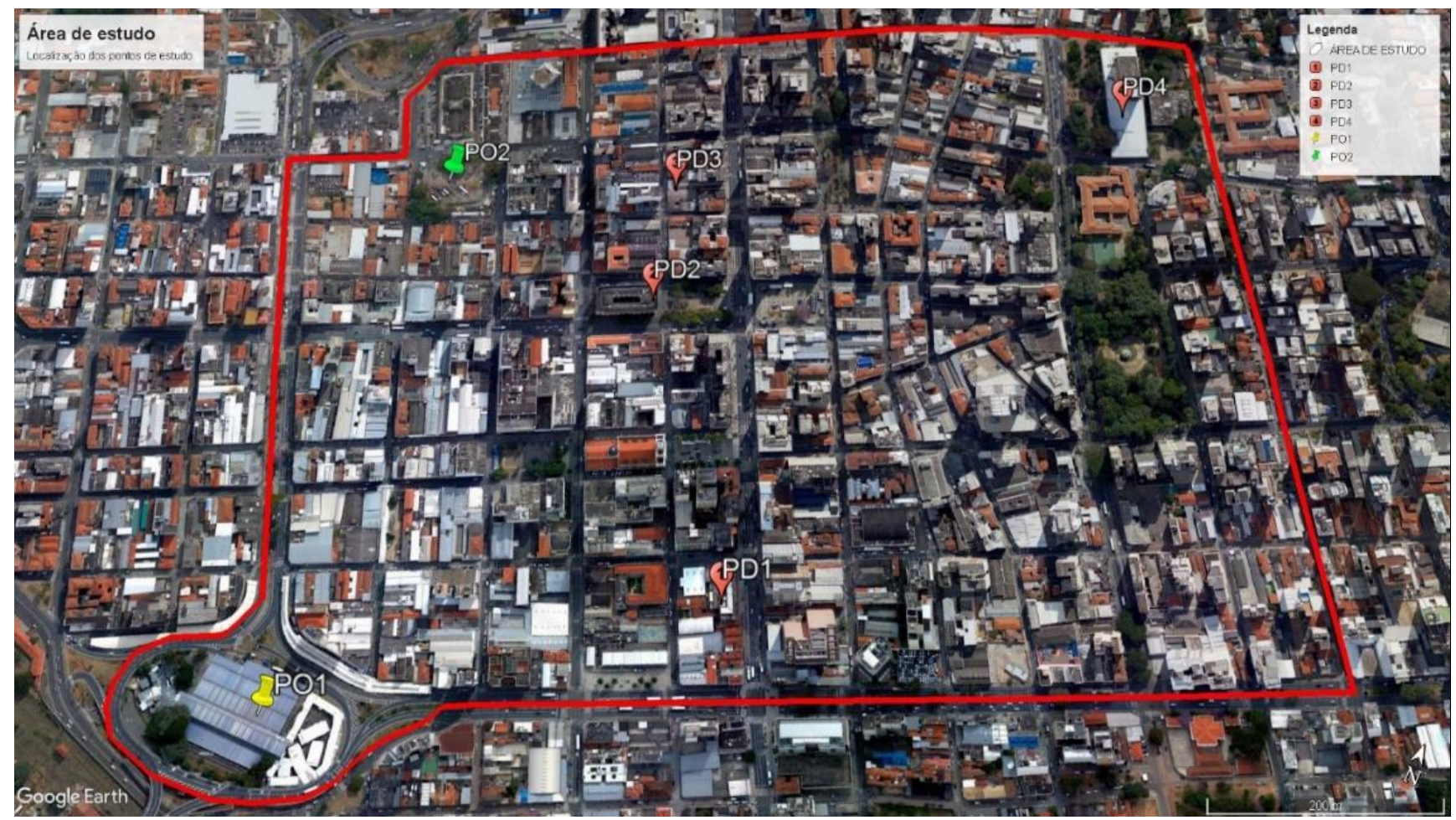

Figura 1 - Especificação dos pontos de interesse para o estudo. Fonte: Adaptado Google Earth Pro, (2020).

Tabela 1 - Especificação dos pontos de interesse

\begin{tabular}{ll}
\hline Pontos & Caracterização \\
\hline PO1 (Ponto de Origem) & Maior e principal terminal da cidade de Campinas. Atende a um total de 32 \\
Terminal Central & linhas de ônibus. \\
PO2 (Ponto de Origem) & Junto ao Mercado Municipal de Campinas, conhecido como Terminal \\
Terminal Mercado & Mercadão. Recebe 28 linhas de ônibus. \\
PD1 (Ponto Destino) & Localizado a cerca de 700 metros do Terminal Central e a 750 metros do \\
Agência de Correios & Terminal Mercado. \\
PD2 (Ponto Destino) & Localizado a cerca de 850 metros do Terminal Central e a 400 metros do \\
Palácio da Justiça & Terminal Mercado. \\
PD3 (Ponto Destino) & Localizado a cerca de 1.400 metros do Terminal Central e a 700 metros do \\
INSS & Terminal Mercado. \\
PD4 (Ponto Destino) & Localizado a cerca de 1.400 metros do Terminal Central e a 700 metros do \\
Prefeitura Municipal & Terminal Mercado. \\
\hline
\end{tabular}

Fonte: Autores (2021).

\section{Matriz Origem-Destino}

A matriz Origem-Destino foi formada por proposições de rotas para caminhada e está associada a um par origem destino na área de estudo. A escolha destas rotas foi feita de forma qualitativa. Para esta pesquisa, cada rota origem-destino foi definida por um conjunto de segmentos. Cada segmento de rota foi delimitado por cruzamentos. A Tabela 2 apresenta exemplos de rotas compostas por segmentos de caminhada na área de estudo. Como exemplo, o segmento S1 liga o Terminal Central (P01) ao início do segmento S2, o segmento S2 liga o fim do segmento 2 ao segmento 3 e assim por diante. 
Tabela 2 - Rotas Origem-Destino

\begin{tabular}{|c|c|c|c|c|c|c|}
\hline Terminal Central (PO1) - Correios (PD1) & $\mathrm{Se}$ & & & & & \\
\hline Rota 1 & S1 & S2 & S3 & S4 & & \\
\hline Rota 2 & S6 & S5 & S2 & S4 & & \\
\hline$\ldots$ & $\ldots$ & $\ldots$ & $\ldots$ & $\ldots$ & ... & $\ldots$ \\
\hline \multicolumn{7}{|l|}{ Terminal Mercado (PO2) - INSS (PD3) } \\
\hline Rota 10 & S8 & S2 & S3 & S5 & & \\
\hline Rota 11 & S5 & S12 & S3 & S5 & & \\
\hline$\ldots$ & $\ldots$ & $\ldots$ & $\ldots$ & $\ldots$ & $\ldots$ & ... \\
\hline
\end{tabular}

Fonte: Autores (2021).

\section{Seleção de Indicadores}

As características associadas a cada segmento de rota foram expressas por seus indicadores. A seleção de indicadores para este estudo se deu a partir dos trabalhos de Bivina e Parida (2019), Carvalho (2018), Silva e Angelis Neto (2019), ITDP Brasil (2018) e Nag et al., (2020). Foram definidos três macro indicadores, subdivididos em outros 12 indicadores, como mostrado na Tabela 3. Indicador é uma medida de ordem qualitativa ou quantitativa, com um significado, que é utilizada para captar e representar informações importantes de elementos que compõem o objeto de observação. É um recurso que permite analisar o aspecto observado a partir da síntese e simplificação de dados complexos (Ferreira, Cassiolato e Gonzalez, 2009).

Tabela 3 - Definição de macro indicadores e indicadores

\begin{tabular}{lll}
\hline Macro Indicador & Indicador & Identificação \\
\hline Ambiente & Largura da calçada & 11 \\
& Avaliação da superfície das calçadas & 12 \\
& Acessibilidade para pessoas com mobilidade & 13 \\
& reduzida & \\
& Participação em rotas & 14 \\
& Acesso ao transporte público & 15 \\
Segurança & Distância & 16 \\
& Iluminação pública & 17 \\
& Cruzamentos & 18 \\
& Largura da via & 19 \\
Conforto & Ocorrência de acidentes & 110 \\
& Atratividade & 111 \\
\hline
\end{tabular}

Fonte: Autores (2021).

Cada segmento foi avaliado de acordo com os diferentes indicadores. 0 macro indicador Ambiente refere-se às características físicas do ambiente da caminhada, sendo dividido e avaliado conforme Tabela 4 . 
Mobilidade em centros urbanos por circuitos de caminhada utilizando o método multicritério PROMETHEE, estudo de caso de Campinas

Tabela 4 - Macro indicador Ambiente e sua avaliação

\begin{tabular}{|c|c|c|}
\hline $\begin{array}{l}\text { Macro } \\
\text { indicador }\end{array}$ & Indicador & Forma de avaliação \\
\hline \multirow[t]{8}{*}{ AMBIENTE } & (I1) Largura das calçadas & $\begin{array}{l}\text { Largura média aproximada em metros das calçadas para o } \\
\text { segmento. }\end{array}$ \\
\hline & $\begin{array}{l}\text { (12)Qualidade das } \\
\text { superfícies das calçadas }\end{array}$ & $\begin{array}{l}\text { Pontuação 3: para calçadas em boas condições, com superfície } \\
\text { regular; }\end{array}$ \\
\hline & & $\begin{array}{l}\text { Pontuação 2: para calçadas regulares, com visível falta de } \\
\text { manutenção, com buracos ao longo do caminho, mas não } \\
\text { frequentes ao longo do segmento; } \\
\text { Pontuação 1: calçadas ruins, com problemas de manutenção } \\
\text { graves, presença frequente de buracos e poças ou trechos sem } \\
\text { pavimentação na calçada. }\end{array}$ \\
\hline & $\begin{array}{l}\text { (13) Acessibilidade para } \\
\text { pessoas com mobilidade }\end{array}$ & $\begin{array}{l}\text { Pontuação 1: quando há travessia em nível com rampas de acesso } \\
\text { para pessoas com mobilidade reduzida; }\end{array}$ \\
\hline & reduzida & $\begin{array}{l}\text { Pontuação 0: para quando não há travessia em nível com rampas } \\
\text { de acesso para pessoas com mobilidade reduzida. }\end{array}$ \\
\hline & $\begin{array}{l}\text { (14) Participação em } \\
\text { diferentes rotas }\end{array}$ & $\begin{array}{l}\text { Somatória de vezes que o mesmo segmento foi utilizado para } \\
\text { diferentes rotas. }\end{array}$ \\
\hline & $\begin{array}{l}\text { (15) Acesso ao transporte } \\
\text { público }\end{array}$ & $\begin{array}{l}\text { Pontuação 1: para a presença de transporte público no segmento } \\
\text { em ao menos um dos lados das calçadas (quando houver dois); } \\
\text { Pontuação 0: para a ausência de transporte público no segmento. }\end{array}$ \\
\hline & (16) Distância & Valor em metros do segmento analisado. \\
\hline
\end{tabular}

Fonte: Autores (2021).

O macro indicador Segurança refere-se à segurança pública e pessoal dos pedestres, Tabela 5. 0 indicador Cruzamentos (I8), trata da intersecção que o pedestre deve atravessar de um segmento a outro. 0 indicador Ocorrência de acidentes (I10), considera o levantamento dos acidentes nos cruzamentos, pois estes são os principais locais de ocorrência de acidentes.

Tabela 5 - Macro indicador Segurança e sua avaliação

\begin{tabular}{|c|c|c|}
\hline $\begin{array}{l}\text { Macro } \\
\text { indicador }\end{array}$ & Indicador & Forma de avaliação \\
\hline SEGURANÇA & $\begin{array}{l}\text { (17) } \\
\text { Iluminação } \\
\text { pública }\end{array}$ & $\begin{array}{l}\text { Pontuação 4: para a rua bem iluminada a noite, e direcionada para } \\
\text { a área de pedestres ao longo de todo o segmento; } \\
\text { Pontuação 3: quando existe iluminação, mas é direcionada para a } \\
\text { via de circulação de veículos; } \\
\text { Pontuação 2: quando a iluminação é direcionada para a } \\
\text { circulação de veículos e há elementos que impedem que a } \\
\text { luminosidade chegue às calçadas, tornando-as escuras; } \\
\text { Pontuação 1: quando o caminho é escuro, não há iluminação ou a } \\
\text { iluminação não está funcionando. }\end{array}$ \\
\hline
\end{tabular}

(Continua) 
Tabela 5 - Macro indicador Segurança e sua avaliação

(18)

Cruzamentos

(19) Largura da via

(110) Ocorrência de acidentes
Pontuação 5: indica intersecções em calçadões, vias exclusivas para pedestres;

Pontuação 4: indica intersecções adequadas com rampas de acesso, faixa de pedestre com demarcação no solo e sinalização semafórica exclusiva para pedestres;

Pontuação 3: indica intersecções com rampas de acesso, faixa de pedestres demarcadas, mas sem semáforos;

Pontuação 2: indica intersecções sem rampas de acesso, ou feitas incorretamente, com faixa de pedestre e sem semáforos;

Pontuação 1: indica inexistência de sinalização adequada, sem rampas, sem faixas demarcadas.

Valor aproximado da largura total da via no segmento analisado em metros.

Valor total de acidentes em UPS (Unidade Padrão de Severidade). Foi utilizado o método UPS que faz uma ponderação dos acidentes de trânsito pela gravidade, sendo peso 1 para acidentes com vítimas, 5 para acidentes com feridos, 13 para vítimas fatais. $\bigcirc$ valor é a somatória do número de acidentes multiplicado pela sua gravidade (DENATRAN, 1987).

Fonte: Autores (2021).

Por fim, o macro indicador Conforto refere-se à atratividade visual e conforto local, Tabela 6.

Tabela 6 - Macro indicador Conforto e sua avaliação

\begin{tabular}{|c|c|c|}
\hline $\begin{array}{l}\text { Macro } \\
\text { indicador }\end{array}$ & Indicador & Forma de avaliação \\
\hline \multirow[t]{2}{*}{ CONFORTO } & (I11) Atratividade & $\begin{array}{l}\text { Pontuação 5: para segmento atrativo para uso exclusivo de } \\
\text { pedestres, "calçadões"; } \\
\text { Pontuação 4: segmento atrativo por contar com jardins, praças e } \\
\text { edifícios bem cuidados, prédios com arquitetura diferenciada, } \\
\text { com lojas e vitrines; } \\
\text { Pontuação 3: segmento com prédios bem conservados e com } \\
\text { arborização que permite sombreamento em alguns trechos do } \\
\text { segmento; } \\
\text { Pontuação 2: segmento pouco atrativo com prédios bem } \\
\text { conservados, mas sem arborização; } \\
\text { Pontuação 1: segmentos com imóveis cercados por muros altos, } \\
\text { prédios mal observados, com indícios de vandalismo, causando } \\
\text { sensação de abandono. }\end{array}$ \\
\hline & $\begin{array}{l}\text { (112) Proteção } \\
\text { contra } \\
\text { intempéries }\end{array}$ & $\begin{array}{l}\text { Pontuação 2: para marquises e/ou toldos para proteção do } \\
\text { pedestre em alguns trechos; } \\
\text { Pontuação 1: quando não há infraestrutura de proteção contra } \\
\text { chuva ou sol. }\end{array}$ \\
\hline
\end{tabular}

Fonte: Autores (2021).

Para outras aplicações pode-se considerar dois outros indicadores: o I13 referentes à "velocidade máxima permitida", que é importante, pois segmentos com altas velocidades inibem a prática da caminhada, entretanto é um dado de difícil aquisição, e o I14, de "inclinação das calçadas", que neste estudo não será considerado, por conta da inclinação todos os segmentos da área de estudo estarem abaixo do mínimo de $5 \%$ e portanto, não interferindo significativamente nos resultados. 


\section{Matriz Segmento-Indicador}

A avaliação de uma rota é resultado da avaliação dos segmentos que a compõem. Neste sentido, a matriz Segmento-Indicador associa valores dos indicadores a cada segmento como na Tabela 7. Com exemplo, a21 é o valor assumido pelo indicador I1 para o segmento 2, a33 é o valor assumido pelo indicador I3 no segmento 3. Este procedimento foi realizado para todos os segmentos.

Tabela 7 - Segmento-Indicador

\begin{tabular}{lllllll}
\hline $\mathbf{N}^{\circ}$ Segmento & Indicador I1 & Indicador $\mathbf{1 2}$ & Indicador $\mathbf{3 3}$ & Indicador $\mathbf{~ 4}$ & Indicador li \\
\hline S1 & a11 & a12 & a13 & al4 & $\ldots$ \\
S2 & a21 & a22 & a23 & a24 & $\ldots$ \\
S3 & a31 & a32 & a33 & a34 & $\ldots$ \\
$\ldots$ & $\ldots$ & $\ldots$ & $\ldots$ & $\ldots$ & $\ldots$ \\
Si & ail & ai2 & ai3 & ai4 & $\ldots$ \\
\hline
\end{tabular}

Fonte: Autores (2021).

\section{Desempenho das rotas}

A partir da composição da matriz Origem-Destino e Segmento-Indicador, foi definido o desempenho total por indicador de cada rota. Os dados para avaliação dos indicadores possuem diferentes fontes, como de órgãos públicos, softwares gratuitos como o Google Earth Pro e o Google Street View, além da participação do decisor. Como exemplo, o indicador "média da largura das calçadas" para a rota X, que utiliza os segmentos S6, S5, S2 e S4 pode ser determinado pela expressão:

$$
I M_{j}\left(R_{x}\right)=\frac{I j(S 6)+I j(S 5)+I j(S 2)+I j(S 4)}{4}
$$

Ou seja:

$$
I M_{j}\left(R_{x}\right)=\frac{a_{6 j}+a_{5 j}+a_{2 j}+a_{4 j}}{4}
$$

Onde $a_{i j}$ é a avaliação do segmento i em relação ao indicador j.

Os indicadores consolidados para cada rota, expressos pela Tabela 8, são considerados como critérios de entrada do método PROMETHEE.

\begin{tabular}{|c|c|c|c|c|}
\hline & & IC 2 & IC .. & IC $n$ \\
\hline $\begin{array}{l}\text { Rota } X \\
\text { Rota } Y\end{array}$ & IM1 & IM2 & IMk & \\
\hline Rota Z & & IM2 & IMk & IMn \\
\hline
\end{tabular}

Tabela 8 - Indicadores Consolidados

Fonte: Autores (2021).

\section{Método multicritério PROMETHEE}

Este trabalho optou pela utilização do método PROMETHEE II por reunir características interessantes para ser utilizado nos processos decisórios de gestões públicas, pois é fácil seu entendimento, potencializando sua transparência no processo decisório, o que é muito importante no setor público (Jannuzzi, Miranda e Silva, 2009). Possui também a vantagem de se encontrar apoio para a sua implementação pela disponibilização de softwares gratuitos como o Visual PROMETHEE (VPSOLUTIONS, 2015). 
Os métodos multicritérios de auxílio à decisão se propõem ao ordenamento completo de um conjunto finito de alternativas da melhor para a pior em problemas com múltiplos critérios conflitantes. Muitas organizações, empresas privadas, administrações públicas, centros de pesquisa e universidades em todo o mundo têm utilizado o método PROMETHEE para tomarem decisões ou avaliar problemas como: bancos, localização de instalações, recursos humanos, recursos hídricos, investimento, química, compras, problemas ambientais, planejamento, gestão de projetos, entre outros (Behzadian et. al, 2010). Seu sucesso é devido às suas propriedades matemáticas e à sua particular facilidade de uso. Outro fator que o torna importante é a possibilidade de considerar indicadores objetivos, isto é, por meio de dados de pesquisas públicas, criando critérios estruturados de avaliação das alternativas para regiões ou grupos sociodemográficos específicos (Jannuzzi, Miranda e Silva, 2009).

O PROMETHEE é uma família de métodos que buscam propor alternativas mais balanceadas, ou seja, alternativas que apresentam um maior desempenho geral médio dos critérios considerados para análise (GOMES, 2007). É um método de apoio à decisão que compara várias alternativas de decisão, e a partir do conceito de superação ou sobreclassificação (De Lima et al., 2014). Sua implementação requer dois tipos de informações: a determinação do peso e a aplicação da função preferência.

A primeira etapa consistiu em atribuir pesos para indicadores de acordo com a importância de cada indicador adotado na análise, o peso de um critério refere-se ao quanto ele é importante em relação aos outros critérios, e deve ser ajustado de acordo com as prioridades do decisores. O PROMETHEE II assume que o tomador de decisão é capaz de pesar os critérios de forma adequada, pelo menos quando o número de critérios não é muito grande (Macharis et al., 2004)

Na segunda etapa, foi definida a função de preferência (ou superação), utilizada para ordenar as alternativas segundo o conceito de dominância, transforma a diferença obtida entre duas alternativas em um grau de preferência que está na faixa entre 0 e 1 . Neste trabalho foi aplicada a metodologia de Brans e Vincke (1986) de fácil aplicação, sendo que, para cada indicador, existe um valor "q" para a indiferença, um valor "p" para a preferência explícita e um valor intermediário "d" entre "p" e "q" que representa a diferença entre duas ações para um determinado indicador, como na Eq.1.

$$
P_{j}(a, b)=\left\{\begin{aligned}
0, & \text { se } d_{j}<q_{j} \\
\frac{d_{j}-q_{j}}{p_{j}-q_{j}}, & \text { se } q_{j}<d_{j}<p_{j} \\
1, & \text { se } d_{j}>p_{j}
\end{aligned}\right.
$$

Os principais passos para a aplicação do método PROMETHEE foram:

- Passo 1: Determinação do desvio - Na aplicação deste estudo representa a comparação de um mesmo indicador da a rota "a "e "rota" b (Eq. 2)

$d_{j}(a, b)=g_{j}(a)-g_{j}(b)$

Onde dj(a,b) é a diferença entre a avaliação da rota a e de b em cada indicador.

- Passo 2: Aplicação da função preferência (Eq. 3),

$P_{j}(a, b)=F_{j}\left[d_{j}(a, b)\right]$

Onde Pj (a,b) denota a preferência da rota "a" em relação a rota "b" em cada indicador, em função de dj(a,b).

- Passo 3: Cálculo da preferência global (Eq. 4), 


$$
\pi(a . b)=\sum_{j=1}^{k} w_{j} \times P_{j}(a, b) \forall a, b \in A
$$

Onde $\pi(a, b)$ de "a" sobre "b" é definido como o a soma ponderada $\mathrm{p}(\mathrm{a}, \mathrm{b})$ para cada indicador, e wj e o peso associado ao j-ésimo indicador.

- Passo 4: Cálculo da classificação parcial (Eq. 5 e 6),

$$
\begin{aligned}
& \phi_{+}(a)=\frac{1}{n+1} \sum_{x \in A} \pi(a, x) \\
& \phi_{-}(a)=\frac{1}{n+1} \sum_{x \in A} \pi(x, a)
\end{aligned}
$$

Onde $\phi+($ a) e $\phi$-(a) são fluxos positivos e negativos para cada rota, respectivamente.

- Passo 5: Cálculo do PROMETHEE ranking completo (Eq. 7),

$$
\phi(a)=\phi_{+}(\mathrm{a})-\phi_{-}(\mathrm{a})
$$

Onde $\phi_{+}(a)$ determina o quanto a rota "a" domina as outras rotas; $\phi_{-}(a)$ determina o quanto outras rotas dominam a rota "a", enquanto $\phi(a)$ denota o fluxo líquido de saída para cada rota. A execução do algoritmo até a Eq.5 do Passo 4 constitui-se no PROMETHEE I e fornece ranking parcial de rotas. 0 PROMETHEE II. Eq. 7, fornece uma classificação completa das rotas, desde as melhores até as piores. A rota classificada em primeiro lugar pode ser considerada como Benchmarking, aquela considerada como referência segundo o conjunto de critérios previamente estabelecidos.

\section{Resultados e Discussões}

A análise das melhores rotas para o centro urbano de Campinas iniciou-se pela determinação de tabelas Origem-Destino. Procurou-se selecionar de três a sete alternativas entre cada origem-destino evitando-se segmentos por grandes avenidas pelo conflito frequente com veículos. Considerou-se na escolha as características do ambiente, do conforto e segurança do pedestre. Destaca-se que rotas para caminhada são bidirecionais.

Como exemplo de aplicação analisa-se a origem-destino entre o Terminal Central (P01) e Correios (PD1). Conjuntos com diferentes segmentos compõem rotas diferentes, como apresentado na Tabela $9 \mathrm{e}$ detalhado na Figura 2. 


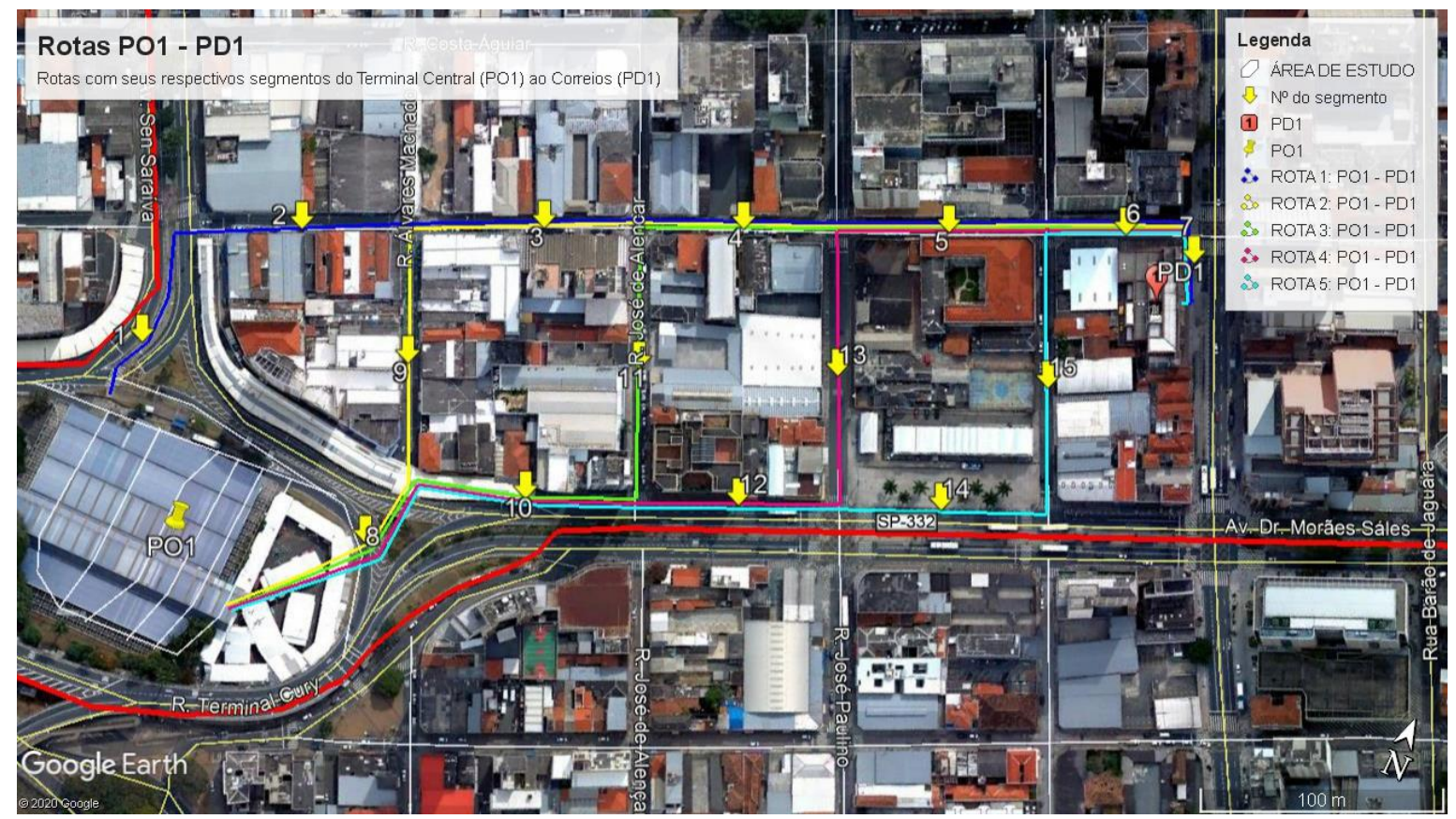

Figura 2 - Rotas PO1 ao PD1. Fonte: Google Earth Pro.

Tabela 9 - Origem-Destino para PO1-PD1

\begin{tabular}{lllllllll}
\hline Terminal Central (PO1) - Correios (PD1) & \multicolumn{7}{l}{ SEGMENTOS } \\
\hline ROTA 1 & 1 & 2 & 3 & 4 & 5 & 6 & 7 \\
ROTA 2 & 8 & 9 & 3 & 4 & 5 & 6 & 7 \\
ROTA 3 & 8 & 10 & 11 & 4 & 5 & 6 & 7 \\
ROTA 4 & 8 & 10 & 12 & 13 & 5 & 6 & 7 \\
ROTA 5 & 8 & 10 & 12 & 14 & 15 & 6 & 7 \\
\hline
\end{tabular}

Fonte: Autores (2021).

Para pequenas distâncias, a coincidência de segmentos é mais frequente, já que a possibilidade de escolha diminui. Foi privilegiada a passagem pelos segmentos 3-4-5-6 em relação aos segmentos 10-12-14 por estes últimos estarem associado a à Avenida. Dr. Moraes Sales, uma avenida das mais movimentadas da região central não sendo favorável à prática da caminhada. Como pode ser visto na Figura 2 a Rota 1 (azul) é composta dos seguintes segmentos: 1, 2, 3, 4, 5, 6, e 7. Cada coluna da tabela Origem-Destino representa uma rota. Assim, a Rota 1 é composta pelos segmentos de 1 a 7, a Rota 2 é composta pelos: 8, 9, 3, 4, 5, 6, e 7, e assim por diante.

Mesmo procedimento deve ser aplicado a todo o conjunto Origem-Destino. No caso de da área de estudo foram formadas nove tabelas Origem-Destino.

\section{Matriz Segmento-Indicador PO1-PDI}

As rotas selecionadas para avaliação entre P01 e PD1 envolvem quinze segmentos, como exposto na Tabela 9. A Tabela Segmento-Indicador associa a cada segmento de uma rota os valores atribuídos a cada indicador, como na Tabela 10. 
Tabela 10 - Segmento-Indicador PO1 ao PDI

\begin{tabular}{cllllllllllll}
\hline PO1 - PD1 & & \multicolumn{1}{c}{ Indicadores } \\
Segmento & 11 & 12 & 13 & 14 & 15 & 16 & 17 & 18 & 19 & 110 & 111 & 112 \\
\hline 1 & 3,8 & 3 & 0 & 1 & 0 & 79,1 & 3 & 3 & 7,1 & 14 & 1 & 0 \\
2 & 1,5 & 3 & 0 & 1 & 0 & 90,9 & 3 & 1 & 6 & 0 & 1 & 0 \\
3 & 1,5 & 3 & 0 & 2 & 0 & 92,6 & 2 & 2 & 6 & 4 & 2 & 1 \\
4 & 1,35 & 3 & 0 & 3 & 0 & 82 & 2 & 2 & 6 & 0 & 2 & 0 \\
5 & 1,48 & 3 & 0 & 4 & 0 & 86,5 & 3 & 2 & 5,8 & 0 & 2 & 0 \\
6 & 1,45 & 3 & 0 & 5 & 0 & 64 & 3 & 4 & 5,8 & 16 & 2 & 0 \\
7 & 3,97 & 3 & 0 & 5 & 1 & 34,5 & 3 & 5 & 12,1 & 0 & 2 & 0 \\
8 & 8,4 & 3 & 1 & 4 & 0 & 105 & 4 & 5 & 0 & 0 & 5 & 1 \\
9 & 8,4 & 3 & 1 & 1 & 0 & 104 & 4 & 5 & 0 & 0 & 5 & 1 \\
10 & 4 & 3 & 0 & 3 & 0 & 98 & 4 & 3 & 8 & 1 & 5 & 1 \\
11 & 1,4 & 3 & 0 & 1 & 0 & 119 & 3 & 2 & 5,8 & 4 & 2 & 0 \\
12 & 4 & 3 & 0 & 2 & 0 & 84,1 & 3 & 3 & 8 & 34 & 2 & 1 \\
13 & 2,88 & 3 & 0 & 1 & 0 & 119 & 3 & 2 & 8,5 & 0 & 2 & 1 \\
14 & 4 & 3 & 0 & 1 & 1 & 86,5 & 4 & 4 & 8 & 20 & 3 & 1 \\
15 & 1,41 & 3 & 0 & 1 & 0 & 119 & 3 & 2 & 5,8 & 0 & 1 & 0 \\
\hline
\end{tabular}

Fonte: Autores (2021).

Como por exemplo, o Segmento 1 apresenta valor 3,8 para o indicador I1, 3 para o I2, zero para o I3, 1 para o I4, zero para o I5, 79,1 para o I6, e assim sucessivamente. 0 indicador I6 indica o comprimento em metros do segmento. Por exemplo, o segmento 1 mede 79,1 m.

\section{Matriz de desempenho das rotas PO1-PDI}

A tabela desempenho das rotas associa os indicadores médios de cada segmento, com as rotas. Um caso particular é o indicador da distância I6 que foi tomado como o somatório das distâncias de todos os segmentos que pertencem a rota. Como exemplo de aplicação, são consideradas as rotas entre o Terminal Central (P01) e os Correios (PD1), Tabela 11.

Tabela 11 - Desempenho das rotas do Terminal Central (PO1) aos Correios (PD1).

\begin{tabular}{llllllllllllll}
\hline PO1 - PD1 & \multicolumn{1}{c}{ Ambiente } & \multicolumn{1}{c}{ Indicadores } \\
& \multicolumn{1}{c}{} & \multicolumn{1}{c}{ Segurança } & \multicolumn{3}{c}{ Conforto } \\
ROTAS & 11 & 12 & 13 & 14 & 15 & 16 & 17 & 18 & 19 & 110 & 111 & 112 \\
\hline ROTA 1 & 2,15 & 3,00 & 0,00 & 3,00 & 0,14 & 529,60 & 2,71 & 2,71 & 6,97 & 4,86 & 1,71 & 0,14 \\
ROTA 2 & 3,79 & 3,00 & 0,29 & 3,43 & 0,14 & 568,60 & 3,00 & 3,57 & 5,10 & 2,86 & 2,86 & 0,43 \\
ROTA 3 & 3,15 & 3,00 & 0,14 & 3,57 & 0,14 & 589,00 & 3,14 & 3,29 & 6,21 & 3,00 & 2,86 & 0,29 \\
ROTA 4 & 3,74 & 3,00 & 0,14 & 3,43 & 0,14 & 591,10 & 3,29 & 3,43 & 6,89 & 7,29 & 2,86 & 0,57 \\
ROTA 5 & 3,89 & 3,00 & 0,14 & 3,00 & 0,29 & 591,1 & 3,43 & 3,71 & 6,81 & 10,14 & 2,86 & 0,57 \\
\hline
\end{tabular}

Fonte: Autores (2021).

\section{Classificação do conjunto de rotas pelo método PROMETHEE}

A classificação segundo o desempenho de uma rota utiliza o software Visual PROMETHEE (VPSOLUTIONS, 2015) na versão PROMETHEE II.

Os primeiros dados de entrada para análise é o estabelecimento dos pesos para cada indicador. Neste estudo foram propostos três cenários para representar a percepção do pedestre, a visão de um órgão público e o terceiro de uma entidade não-governamental. 0 Cenário 1 priorizou as calçadas, com pontuação máxima para largura e avaliação da superfície, cruzamentos e menor distância. 0 Cenário 2 buscou 
equilibrar todos os itens. O Cenário 3 priorizou a acessibilidade para pessoas com mobilidade reduzida, iluminação pública, cruzamentos e atratividade dos segmentos. A atribuição para cada indicador varia entre 0 (sem importância) a 5 (muito importante). Após a atribuição dos pesos em cada cenário, foi feita uma média para cada indicador, Tabela 12. Os pesos finais são resultantes da divisão do valor do indicador médio de cada cenário, coluna 5 , pela soma dos indicadores médios de todos os cenários. Ou seja, o valor da coluna 4 dividido pela soma de todos os elementos da coluna 4, que é 43,7.

Tabela 12 - Definição dos pesos dos indicadores

\begin{tabular}{|c|c|c|c|c|c|}
\hline \multirow[b]{2}{*}{ Indicadores } & \multicolumn{5}{|l|}{ Cenários } \\
\hline & Cenário 1 & Cenário 2 & Cenário 3 & Média & Pesos \\
\hline Largura da calçada (I1) & 5 & 4 & 3 & 4 & 0,09 \\
\hline Avaliação da superfície das calçadas (I2) & 5 & 3 & 4 & 4 & 0,09 \\
\hline Acessibilidade para pessoas com mobilidade reduzida (I3) & 3 & 4 & 5 & 4 & 0,09 \\
\hline Participação em rotas (14) & 2 & 5 & 3 & 3,3 & 0,08 \\
\hline Acesso ao transporte público (I5) & 3 & 2 & 4 & 3 & 0,07 \\
\hline Distância (l6) & 5 & 4 & 3 & 4,0 & 0,09 \\
\hline Iluminação pública (I7) & 2 & 2 & 5 & 3,0 & 0,07 \\
\hline Cruzamentos (I8) & 5 & 4 & 5 & 4,7 & 0,11 \\
\hline Largura da via (19) & 2 & 2 & 3 & 2,3 & 0,05 \\
\hline Ocorrência de acidentes (I1 1) & 5 & 4 & 4 & 4,3 & 0,10 \\
\hline Atratividade (I12) & 4 & 4 & 5 & 4,3 & 0,10 \\
\hline \multirow[t]{2}{*}{ Proteção contra intempéries (I13) } & 4 & 2 & 2 & 2,7 & 0,06 \\
\hline & \multicolumn{3}{|l|}{ Somatória } & 43,7 & 1,00 \\
\hline
\end{tabular}

Fonte: Autores (2021).

Alguns indicadores devem ser maximizados e outros minimizados como visto na Tabela 13, entendendo que quanto maior forem os indicadores I1, I2, I3, I4, I5, I7, I8, I11 e I12, maior o incentivo para a prática da caminhada. Em contrapartida, quanto menor forem os indicadores I6, I9, e I10, mais influenciam à prática da caminhada.

Tabela 13 - Preferências máximas e mínimas de cada indicador

\begin{tabular}{lllllllllllll}
\hline & \multicolumn{2}{c}{ Indicadores } & & & & & & & & & & \\
& $\mathbf{1 1}$ & $\mathbf{1 2}$ & $\mathbf{1 3}$ & $\mathbf{1 4}$ & $\mathbf{1 5}$ & $\mathbf{1 6}$ & $\mathbf{1 7}$ & $\mathbf{1 8}$ & $\mathbf{1 9}$ & $\mathbf{1 1 0}$ & $\mathbf{1 1 1}$ & $\mathbf{1 1 2}$ \\
\hline Min/Max & Max & Max & Max & Max & Max & Min & Max & Max & Min & Min & Max & Max \\
Peso & 0,09 & 0,09 & 0,09 & 0,08 & 0,07 & 0,09 & 0,07 & 0,11 & 0,05 & 0,10 & 0,10 & 0,06 \\
\hline
\end{tabular}

Fonte: Autores (2021).

O limite de indiferença "q" que é o maior desvio considerado insignificante na comparação de duas rotas foi definido como $5 \%$ da diferença entre o maior valor e o menor valor para cada indicador.

Os dados de entrada para PROMETHEE GAIA II, contendo cinco rotas entre o Terminal Central (P01) e os Correios (PD1), a diferença entre o maior e menor valor de cada indicador médio, e "q" e "p" estão apresentados na Tabela 14. Estas rotas foram escolhidas a partir da análise dos segmentos dentro do espaço considerado. 
Tabela 14 - Definição parâmetros PO1 - PDI

\begin{tabular}{|c|c|c|c|c|c|c|c|c|c|c|c|c|}
\hline \multirow[b]{2}{*}{ PO1 - PD1 } & \multicolumn{12}{|c|}{ Indicadores } \\
\hline & 11 & 12 & 13 & 14 & 15 & 16 & 17 & 18 & 19 & 110 & 111 & 112 \\
\hline Mínimo/Máximo & MAX & MAX & MAX & MAX & MAX & MIN & MAX & MAX & MIN & MIN & MAX & MAX \\
\hline Pesos & 0,09 & 0,09 & 0,09 & 0,08 & 0,07 & 0,09 & 0,07 & 0,11 & 0,05 & 0,10 & 0,10 & 0,06 \\
\hline ROTA 1 & 2,15 & 3,00 & 0,00 & 3,00 & 0,14 & 529,6 & 2,71 & 2,71 & 6,97 & 4,86 & 1,71 & 0,14 \\
\hline ROTA 2 & 3,79 & 3,00 & 0,29 & 3,43 & 0,14 & 568,6 & 3,00 & 3,57 & 5,10 & 2,86 & 2,86 & 0,43 \\
\hline ROTA 3 & 3,15 & 3,00 & 0,14 & 3,57 & 0,14 & 589,0 & 3,14 & 3,29 & 6,21 & 3,00 & 2,86 & 0,29 \\
\hline ROTA 4 & 3,74 & 3,00 & 0,14 & 3,43 & 0,14 & 591,1 & 3,29 & 3,43 & 6,89 & 7,29 & 2,86 & 0,57 \\
\hline ROTA 5 & 3,89 & 3,00 & 0,14 & 3,00 & 0,29 & 591,1 & 3,43 & 3,71 & 6,81 & 10,1 & 2,86 & 0,57 \\
\hline Maior valor/ Menor valor & 1,74 & 0,00 & 0,29 & 0,57 & 0,14 & 61,50 & 0,71 & 1,00 & 1,87 & 7,29 & 1,14 & 0,43 \\
\hline Indiferença "q" de 5\% & 0,09 & 0,00 & 0,01 & 0,03 & 0,01 & 3,08 & 0,04 & 0,05 & 0,09 & 0,36 & 0,06 & 0,02 \\
\hline Preferência "p" de $20 \%$ & 0,35 & 0,00 & 0,06 & 0,11 & 0,03 & 12,30 & 0,14 & 0,20 & 0,37 & 1,46 & 0,23 & 0,09 \\
\hline
\end{tabular}

Fonte: Autores (2021).

A Tabela 15 apresenta o resultado da classificação das rotas, como resultado da aplicação do PROMETHEE GAIA II,

Tabela 15 - Classificação final PO1 - PD1

\begin{tabular}{ccc}
\hline Classificação & Rota & Fluxo de dominância líquida \\
\hline $1^{\circ}$ & 2 & 0,3516 \\
$2^{\circ}$ & 5 & 0,1332 \\
$3^{\circ}$ & 4 & 0,0273 \\
$4^{\circ}$ & 3 & 0,0235 \\
$5^{\circ}$ & 1 & $-0,5356$ \\
\hline
\end{tabular}

Fonte: Autores, (2021).

A Rota 2 apresentou melhor pontuação por obter valor mais elevado no indicador acessibilidade de pessoas com mobilidade reduzida (I3), menor valor no indicador ocorrência de acidentes (I10) e maior valor no indicador atratividade para a circulação de pedestres (I11), que possuem os pesos mais elevados. Apresenta também o melhor desempenho no indicador largura da rua (I9).

A partir da identificação da melhor rota entre os terminais rodoviários urbanos e os pontos mais relevantes de prestação de serviços, apresentada na Tabela 16, é sugerida a ocupação do espaço urbano do centro da cidade de Campinas para a caminhada.

Tabela 16 - Rotas Origem-Destino

\begin{tabular}{lll}
\hline Origem-Destino & Rota & $\begin{array}{l}\text { Fluxo de dominância } \\
\text { líquido }\end{array}$ \\
\hline PO1 - PD1: Terminal Central - Correios & ROTA 2 & 0,3516 \\
PO1 - PD2: Terminal Central - Palácio da Justiça & ROTA 9 & 0,5353 \\
PO1 - PD3: Terminal Central - INSS & ROTA 14 & 0,5403 \\
PO1 - PD4: Terminal Central - Prefeitura & ROTA 22 & 0,4375 \\
PO2 - PD1: Terminal Mercado - Correios & ROTA 26 & 0,2420 \\
PO2 - PD2: Terminal Mercado - Palácio da Justiça & ROTA 32 & 0,0920 \\
PO2 - PD3: Terminal Mercado - INSS & ROTA 35 & 0,2100 \\
PO2 - PD4: Terminal Mercado - Prefeitura & ROTA 39 & 0,1354 \\
PO1 - PO2: Terminal Central - Terminal Mercado & ROTA 44 & 0,3360 \\
\hline
\end{tabular}


Esta ocupação, considerando nove rotas, envolve 47 segmentos., como apresentado na Figura 3.

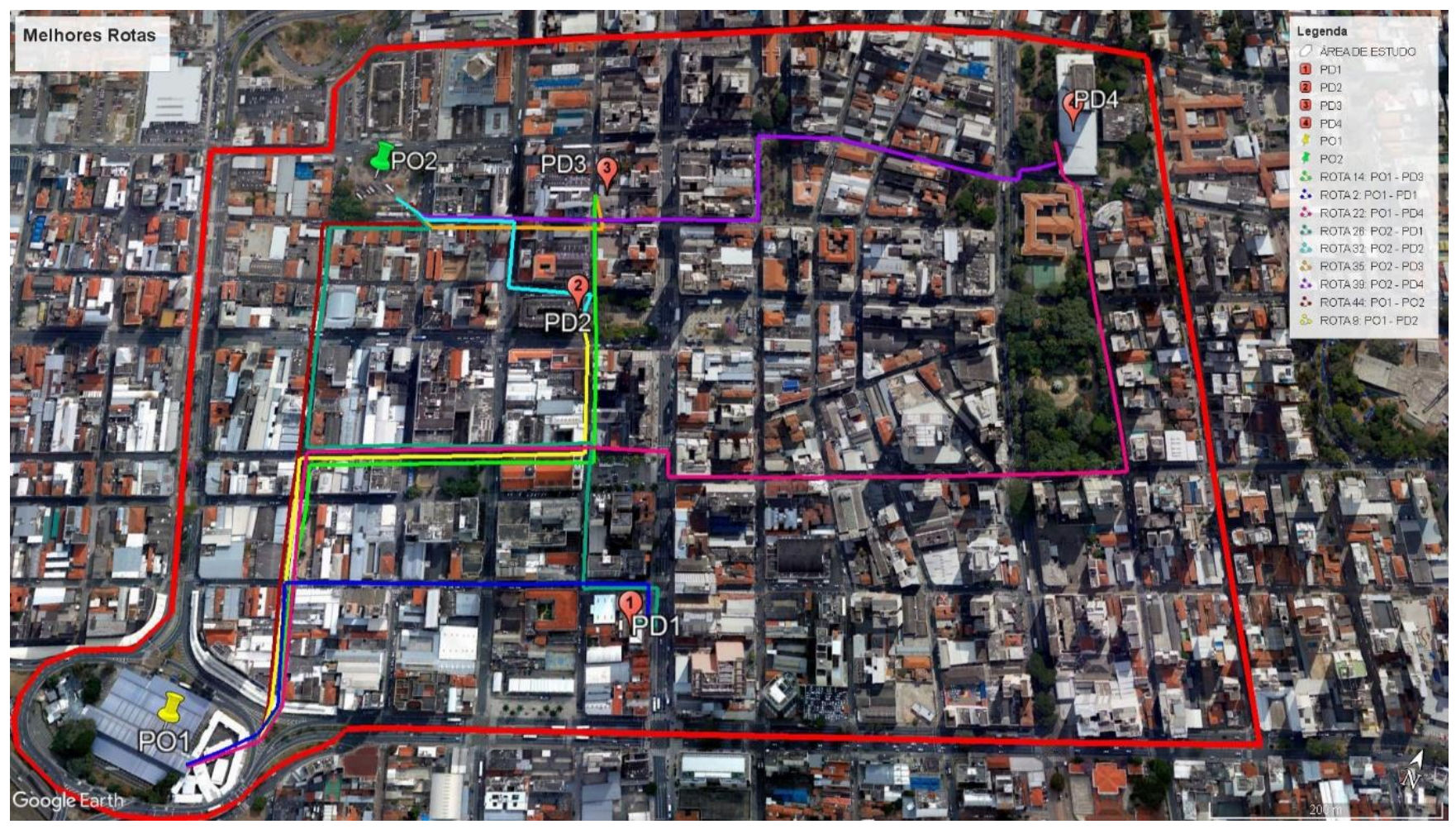

Figura 3 - Melhores rotas. Fonte: Google Earth Pro (2020).

\section{Considerações finais}

Incentivar a integração do transporte público com a caminhada pode ser uma abordagem benéfica para a saúde do cidadão e um meio de acender a questões sociais e ambientais em um meio urbano principalmente em um país em desenvolvimento como o Brasil. Entretanto, as pessoas que se deslocam por caminhada possuem diferentes capacidades de percepção e agilidade associadas ao sexo, idade e limitações físicas, devem realizar um esforço físico e estão em contato direto com o entorno. Reconhecendo a importância desta atividade em um centro urbano, os formuladores de políticas públicas precisam atender às necessidades e expectativas do pedestre, fornecendo uma adequada infraestrutura para uma caminhada segura e confortável.

A contribuição deste trabalho está em propor que a conectividade entre terminais públicos urbanos e pontos de prestação de serviços essenciais ao cidadão seja considerada pelo planejador urbano a partir de determinação de rotas que considerem as características do ambiente pelo qual o pedestre vai percorrer, $o$ conforto e a segurança da caminhada. A escolha da melhor entre as possíveis rotas interligando uma origem e destino foi formulada como um problema multicritério e utilizado o Método Promethee II para sua solução. Para a aplicação do método foram especificados sete de pontos de interesse no centro urbano de Campinas e selecionados indicadores para avaliação da adequação dos segmentos de espaços públicos (ruas calçadões, praças) a prática da caminhada. As características destes espaços foram pontuadas por meio de macro três macros indicadores: ambiente, segurança e conforto decompostos e 12 indicadores. Foi atribuído um peso a cada um destes indicadores para representar a percepção do usuário e do setor público. Estes pesos foram estabelecidos a partir três diferentes cenários, cada um privilegiando um dos três macro indicadores. A seguir foi aplicada a comparação par a par de cada rota para cada conjunto origem destino que resultou na escolha de melhor rota entre cada ponto de origem (terminal rodoviário) e ponto de destino (ponto relevantes de prestação de serviços). Por fim, foi sugerida uma configuração, a partir da composição das nove melhores rotas para todo conjunto origem destino, para o centro urbano de Campinas. 
A implementação das soluções aqui sugeridas depende de apoio do poder público que deve avaliá-las e implementá-las, ao mesmo tempo desenvolver ações que contribuam para um trânsito tranquilo, calçadas adequadas nos segmentos de rotas escolhidos, fiscalização para evitar o uso inadequado do espaço público, colocações de bancas, marcação de travessias de pedestre.

Com a proposição desta metodologia para avaliação do espaço público pode-se também avaliar o impacto de outras medidas, como a implantação de novos calçadões e conceito de ruas compartilhadas. Assim, como continuidade deste trabalho propõe-se a interferência no ambiente atual pelo poder público com a implementação de espaços compartilhados entre pedestres, bicicletas e veículos motorizados como meio de tornar o centro urbano mais atrativo.

\section{Agradecimentos}

Este estudo foi financiado em parte pela Coordenação de Aperfeiçoamento de Pessoal de Nível Superior - Brasil (CAPES) - Código Financeiro 001.

\section{Declaração de disponibilidade de dados}

O conjunto de dados que dá suporte aos resultados deste artigo está disponível no SciELO DATA e pode ser acessado em https://doi.org/10.48331/scielodata.WRT406.

\section{Referências}

Associação Nacional de Transportes Públicos - ANTP. (2018). Sistema de Informações da Mobilidade Urbana da Associação Nacional de Transportes Público - SIMOB/ANTP: Relatório geral 2018. Recuperado em 4 de setembro de 2020, de http://files.antp.org.br/simob/simob-metodologia-v11.pdf

Asadi-Shekari, Z., Moeinaddini,M., Aghaabbasi, M., Cool, M., \& Shah, M. S. (2019). Exploring effective micro-level items for evaluating inclusive walking, facilities on urban streets (applied in Johor Bahru, Malaysia). Sustainable Cities and Society, 49. doi.org/10.1016/j.scs.2019.101563.

Asadi-Shekari, Z.; Moeinaddini, M. \& Aghaabbasi, M. (2014). A pedestrian level of service method for evaluating and promoting walking facilities on campus streets, Land Use Policy 38, 175-193. doi: 10.1016/j.landusepol.2013.11.007

Behzadian, M., Kazemzadeh, R.B. \& Albadvi, A. (2010). PROMETHEE: A comprehensive literature review on methodologies and applications. European Journal of Operational Research 200,198-215. doi.org/10.1016/j.ejor.2009.01.021.

Bloomberg, M. R., \& Burden, A. M. (2006). New York City Pedestrian Level of Service Study Phase I. NYC Department of City Planning, 2006. Recuperado em 03 de setembro de 2020, de https://www1.nyc.gov/assets/planning/download/pdf/plans/transportation/td_fullpedlosb.pdf.

Brans, J. P., Vincke, Ph. \& Mareschal, B. (1986). How to select and how to rank projects: The PROMETHEE method. European Journal of Operational Research 24(2), 228-238. doi.org/10.1016/0377-2217(86)90044-5

Brasil. (2001, 10 de julho). Lei 10.257 de 10 de julho de 2001. Estatuto da Cidade. Brasília-DF.

Brasil. (2012, 3 de janeiro). Lei no 12.587, de 3 de janeiro de 2012. Institui as diretrizes da Política Nacional de Mobilidade Urbana. Brasília-DF.

Bivina, G. R., \& Parida, M. (2019). Modelling perceived pedestrian level of service of sidewalks: A structural equation approach. Transport, 34(3), 339-350. doi.org/10.3846/transport.2019.9819

Carvalho, I. R. V. de. (2018). Caminhabilidade Como Instrumento De Mobilidade Urbana. (Tese de doutorado). Universidade Federal de Minas Gerais, Belo Horizonte.

Clifton, K. J., Singleton, P. A., Muhs, D., \& Schneider, R. (2016). Representing pedestrian activity in travel demand models: Framework and application. Journal of Transport Geography, 52, 11. doi.org/10.1016/j.jtrangeo.2016.03.009

Silva, O. H., \& Angelis Neto, G. D. A. (2019). Índice de Serviço das Calçadas (ISC). Ambiente Construído, 19(1), $221-236$. doi.org/10.1590/s1678-86212019000100303 
De Lima, J. D., Jucá, J. F. T., Reichert, G. A., \& Firmo, A. L. B. (2014). Uso de modelos de apoio à decisão para análise de alternativas tecnológicas de tratamento de resíduos sólidos urbanos na Região Sul do Brasil. Engenharia Sanitaria e Ambiental, 19(1), 33-42. https://doi.org/10.1590/S1413-41522014000100004.

DENATRAN - Departamento Nacional de Trânsito (1987) Manual de Identificação análise e tratamento de pontos negros, $2^{\text {a }}$ ed., 127p, Brasília - DF.

Empresa Municipal de Desenvolvimento de Campinas - EMDEC. (2010a). Terminais Urbanos. Terminal Central. Recuperado em 30 de dezembro de 2020, de www.emdec.com.br/eficiente/sites/portalemdec/pt-

br/site.php?secao=terminais_urbanos\&pub=3298

Empresa Municipal de Desenvolvimento de Campinas - EMDEC. (2010b). Terminais Urbanos. Terminal Mercado. Recuperado em 30 de dezembro de 2020, de www.emdec.com.br/eficiente/sites/portalemdec/ptbr/site.php?secao=terminais_urbanos\&pub=3301

Ernawati, J. (2016). Dimensions Underlying Local People's Preference of Street Characteristics for Walking, Procedia Social and Behavioral Sciences, 234, 461-469. 10.1016/j.sbspro.2016.10.264

Ferreira, H., Cassiolato, M. \& Gonzalez, R. (2009). Uma experiência de desenvolvimento metodológico para avaliação de programas: o modelo lógico do programa segundo tempo. Texto para discussão 1369. Brasília: IPEA.

Gomes, L. F. A. M. (2007). Teoria da Decisão. São Paulo: Thomson Learning.

Instituto Brasileiro de Geografia e Estatística - IBGE. (2010). Censo Demográfico 2010. Rio de Janeiro: IBGE. Recuperado em 11 de maio de 2019, de www.ibge.gov.br

Instituto de Políticas de Transporte \& Desenvolvimento - ITDP BRASIL. (2018). Índice de caminhabilidade. Ferramenta Versão 2.0. Brasil. 66p.

Jannuzzi, P. de M., Miranda, W. L. de, \& Silva, D. S. G. da. (2009). Artigo Políticas Públicas: Aspectos Metodológicos, Aplicativo Operacional e Aplicações. Informática Pública, 11, 69-87. doi.org/10.19094/contextus.v16i3.39904

Krizek, K. J., Forsyth, A., \& Baum, L. (2009). Walking and cycling international literature review: Final report. In Department of Transport Victoria Australia. Recuperado em 10 de novembro de 2020, de https://pdfs.semanticscholar.org/e552/b9f029b5f03842854c43e092d10f7581654d.pdf.

Macharis, C., Springael, J., De Brucker, K. \& Verbeke, A. (2004). PROMETHEE and AHP: The design of operational synergies in multicriteria analysis. Strengthening PROMETHEE with ideas of AHP. European Journal of Operational Research, 153, 307-317. 10.1016/S0377-2217(03)00153-X

Magagnin, R. C. (2009). Cidades Acessíveis: O Planejamento da Infraestrutura para a Circulação de Pedestres. Arquitetura e Urbanismo: novos desafios para o século XXI. 16p.

Malatesta, M. E. B. (2007). Andar a pé: Um modo de Transporte para a Cidade de São Paulo. 2007. 254 f. (Dissertação de Mestrado). Universidade de São Paulo, São Paulo.

Nag, D., Goswami, A. K., Gupta, A., \& Sen, J. (2020). Assessing urban sidewalk networks based on three constructs: a synthesis of pedestrian level of service literature. Transport Reviews, 40(2), 204-240.

https://doi.org/10.1080/01441647.2019.1703841

Oliveira, R. A. de. (2014). Avaliação Do Nível De Serviço Das Calçadas: Estudo De Caso Município De Foz Do Iguaçu-PR (Dissertação de Mestrado). Universidade Federal de Santa Catarina, Florianópolis.

Plano de Mobilidade Urbana de São Paulo - PlAnMOB, (2015). Plano de Mobilidade Urbana do Município de São Paulo 2015. Recuperado em 22 de março de 2020, de http://files.antp.org.br/2016/3/2/plano-mun-mob-urbana-sp-2015_1.pdf

Pucher, J., Dill, J., \& Handy, S. (2010). Infrastructure, programs, and policies to increase bicycling: An international review, Preventive Medicine, 50, S106-S125. 10.1016/j.ypmed.2009.07.028

Silva, O. H., \& De Angelis Neto, G. (2019). Índice de Serviço das Calçadas (ISC), Ambiente Construído. 19(1), $221-236$.

Tian, G. \& Ewing, R. (2017). Transportation Research Part D: Transport and Environment, 52, 340-353.

doi.org/10.1016/j.trpro.2019.07.046

Valentini, L.M., Kuhn, D. \& Vargas, J. C. (2017). Mobilidade Urbana Saudável. I Simpósio Nacional de Gestão e Engenharia Urbana. p. 17. 
Velozo, T. R. V. (2019). Estudo de metodologias para avaliação de calçadas em bairros de Niterói, RJ. Mobilize Brasil. Recuperado em 21 de janeiro de 2021, de https://www.mobilize.org.br/estudos/445/estudo-de-metodologias-paraavaliacao-de-calcadas-em-bairros-de-niteroi-rj.html.

Visual Promethee Solutions - VPSOLUTIONS. (2015). Visual PROMETHEE (Visual PROMETHEE 1.5; p. 64). Recuperado em 4 de setembro de 2020, de www.promethee-gaia.net/assets/vpgetstarted.pdf

Yamamoto, A. M. (2017). Proposição de Medidas para Incentivar as Viagens a Pé na Área Central de São Paulo. (Dissertação de Mestrado). Universidade Estadual de Campinas, Campinas.

Editor responsável: Fábio Duarte

Recebido: 25 mar. 2021

Aprovado: 25 jun. 2021 